\title{
Oscillatory motions observed in eruptive filaments
}

\author{
K. Bocchialini ${ }^{1}$, F. Baudin ${ }^{1}$, S. Koutchmy ${ }^{2}$, G. Pouget ${ }^{1}$, and J. Solomon ${ }^{1}$ \\ ${ }^{1}$ Institut d'Astrophysique Spatiale, Bât. 121, UMR 8617, CNRS/Univ. Paris-Sud, 91405 Orsay, France \\ e-mail: karine.bocchialini@ias.u-psud.fr \\ 2 Institut d'Astrophysique de Paris, UMR 7095, CNRS/UPMC, 98bis boulevard Arago, 75014 Paris, France
}

Received 16 December 2010 / Accepted 6 July 2011

\begin{abstract}
Context. The origin of the variable component of the solar wind is of great intrinsic interest for heliophysics and spaceweather, e.g. the initiation of coronal mass ejections and the problem of mass loss of all stars. It is also related to the physics of coronal neutral sheets and streamers, which occur above lines of magnetic polarity reversal. Filaments and prominences correspond to the cool coronal component of these regions.

Aims. We examine the dynamical behaviour of these structures where reconnection and dissipation of magnetic energy in the turbulent plasma are occurring. The link between the observed oscillatory motions and the eruption occurrence is investigated in detail for two different events.

Methods. Two filaments were analysed using two different datasets: time series of spectra using a transition region line (He I at $584.33 \AA$ ) and a coronal line (Mg X at 609.79 $\AA$ ) measured with CDS on-board SOHO, observed on May 30, 2003, and time series of intensity and velocity images from the NSO/Dunn Solar Telescope in the $\mathrm{H} \alpha$ line on September 18, 1994 for the other. The oscillatory content was investigated using Fourier transform and wavelet analysis and compared to different models.

Results. In both filaments, oscillations are clearly observed, in intensity and velocity in the $\mathrm{He} \mathrm{I}$ and $\mathrm{Mg} \mathrm{X}$ lines, in velocity in $\mathrm{H} \alpha$, with similar periods from a few minutes up to $80 \mathrm{~min}$, with a main range from 20 to $30 \mathrm{~min}$, simultaneously with eruptions. Both filaments exhibit vertical oscillating motions. For the filament observed in the UV (He I and $\mathrm{Mg} \mathrm{X}$ lines), we provide evidence of damped velocity oscillations, and for the filament observed in the visible ( $\mathrm{H} \alpha$ line), we provide evidence that parts of the filament are oscillating, while the filament is moving over the solar surface, before its disappearance.
\end{abstract}

Key words. Sun: filaments, prominences - Sun: oscillations - Sun: atmosphere

\section{Introduction}

Prominences (observed above the limb) and filaments (observed on the solar disc) are complex structures of cool, dense plasma embedded in the two orders of magnitude less dense corona, which is also two orders of magnitude hotter. They are maintained in dynamical equilibrium against gravity by the magnetic field, and they can channel waves propagating outward from the low chromosphere. Filaments and prominences form in both active and quiet-Sun regions. Filaments and prominences that have been stable for weeks can suddenly erupt (e.g. Oliver 2009). Some of the erupting filaments, when heated and becoming visible in coronal emission lines, do not produce a CME: the filament reforms after cooling owing to radiative losses. Such an example was reported by Filippov \& Koutchmy (2002) who discuss the possible origin of the heating related to the dynamical behaviour of the filament, including oscillations.

As pointed out by Isobe \& Tripathi (2006) and Tripathi et al. (2009), oscillations of erupting filaments provide an alternative tool for probing the onset mechanisms of eruptions. SOHO/SUMER observations of a prominence oscillation were discussed in the context of the physics of CMEs (Chen et al. 2008). It was suggested that repetitive reconnections between emerging flux and the pre-existing magnetic field are the trigger mechanism of Doppler velocity oscillations leading to the eruption. Periods near $20 \mathrm{~min}$, lasting four hours before the eruption, were detected. However, much of the literature deals only with quiescent filaments or prominences. The oscillations are classified in two groups based on their amplitude: large amplitudes of several tens of $\mathrm{km} \mathrm{s}^{-1}$ (Ramsey \& Smith 1966) and small amplitudes of a few $\mathrm{km} \mathrm{s}^{-1}$ or less (e.g. Terradas et al. 2002; Oliver \& Ballester 2002; Engvold 2008). Large-amplitude oscillations in filaments, typically $20 \mathrm{~km} \mathrm{~s}^{-1}$ or more, are rare events (Tripathi et al. 2009). They are due to flares or to local emergence of magnetic flux near the filament, while small-amplitude oscillations do not affect the whole structure at one time but are local in nature. Intermediate values are also reported (e.g. Bocchialini et al. 2001) with velocities up to around $13 \mathrm{~km} \mathrm{~s}^{-1}$.

The oscillations in quiescent prominences are classified into three categories traditionally measured from their Doppler signal: short-period (less than $5 \mathrm{~min}$ ), intermediate periods (in the range of 6 to $20 \mathrm{~min}$ ) detected in individual threads of prominences, and long-period (between 40 to $90 \mathrm{~min}$ ) detected over the whole prominence or filament (Molowny-Horas et al. 1997; Oliver \& Ballester 2002; Engvold 2008; Mackay et al. 2010). Ning et al. (2009) report threads in prominences exhibiting vertical and horizontal motions from Hinode/SOT observations close to the $\mathrm{H} \alpha$ line centre at $6563+0.076 \AA$, and the predominant period of the small-amplitude velocity oscillations is about five minutes. Berger et al. (2010) have recently reported smallscale turbulent upflows in quiescent prominences observed with Hinode/SOT, in the Ca II H line at $3969 \AA$ and the $\mathrm{H} \alpha$ line at $6563 \AA$. The recurrence time of these upflows is roughly five 
to eight minutes, and the location remains active on timescales from tens of minutes up to several hours.

Isobe \& Tripathi (2006) and Isobe et al. (2007) report the oscillatory motion of a polar crown filament, observed at $195 \AA$ at a 12 min cadence and in $\mathrm{H} \alpha$, with a long and constant period of about two hours: $5 \mathrm{~km} \mathrm{~s}^{-1}$ amplitude oscillations, measured on the plane of the sky, are detected in 195 - $\AA$ images, and occurred during the slow rise of the filament, which erupted after three periods. Pinter et al. (2008) observed the same filament using the same EUV dataset and wavelet spectra to extract intensities, which show that the filament oscillates as a rigid body, with a period of about $2.5-2.6 \mathrm{~h}$, which is constant along the filament. All the above analysis does not reveal any signature of the eruption. Recent detection of long and ultra-long period oscillations of up to several hours have been reported. Foullon et al. (2004) report the first detection of 8 to $27 \mathrm{~h}$ period in oscillatory intensity variations of a quiescent filament observed at $195 \AA$. The dominant period is $12.1 \mathrm{~h}$. Pouget et al. (2006) report five to six hour period oscillations detected in the Doppler velocity of a quiescent filament, in He I at $584.33 \AA$. Foullon et al. (2009) show that the long period detected in a quiescent filament, using $195 \AA$ images, increases prior to its eruption.

Small-amplitude oscillations have been extensively modelled over the past 20 years in parallel with the development of coronal seismology (Ballester 2006), which aims to interpret the oscillations detected in the corona and its structures in terms of MHD waves as a tool for diagnosing those structures. However, most of these MHD models of prominence oscillations deal with stable filaments and cannot predict their eruption. Vrsnak (1990) developed a simple model of cylindrical prominences to study their eruptive instability and provided a scenario for the eruption onset in terms of mass loss, increase in the electric current caused by slow reconnection below the filament, and twisting motions in the footpoints. Indeed, Koutchmy et al. (2008) observed such behaviour in a prominence just before it erupted and gave rise to a CME, compatible with the standard model (Priest \& Forbes 2000), which invoke resistive instabilities. Filippov \& Koutchmy (2008) propose a scenario where the slow rising of a filament is interpreted as an increase in the non-potentiality of the prominence magnetic field inside the filament before its eruption, when compared to the potential field in the surrounding corona, computed from the observed surface fields.

Several authors (e.g. Wiehr et al. 1989; Molowny-Horas et al. 1998; Terradas et al. 2002; Vršnak et al. 2007; Ning et al. 2009) have discussed damped oscillations in quiescent filaments, disappearing after a few periods, i.e. quickly damped by one or several mechanisms (Oliver 2009). Arregui \& Ballester (2010) review the theoretical damping mechanisms in quiescent prominences as thermal effects, through non-adiabatic processes, mass flows, resonant damping, and partial ionization effects. Vrsnak et al. (1990) report damped post-eruptive oscillations in an active prominence, but clear observations of prominences at limb during their eruption are extremely rare (Berger et al. 2010). We focus here on the analysis of two active filaments, observed during their eruption, from two sets of observations performed at high cadence: one with the Coronal Diagnostic Spectrometer (CDS) (Harrison et al. 1995) on-board SOHO (Fleck et al. 1995) and one with the NSO/Dunn Solar Telescope (DST). These data sets are complemented by observations from the extreme UV imager EIT on-board SOHO and the soft X-ray telescope (SXT) onboard Yohkoh. The two data sets are thus quite different: one consists of spectroscopic observations in the UV (SOHO/CDS), the other of narrow band filtergrams taken in the $\mathrm{H} \alpha$ line (DST).
Table 1. CDS observational parameters.

\begin{tabular}{lc}
\hline \hline Observed Lines & $\operatorname{He~I}(584.33 \AA)$ \\
& $\operatorname{Mg~X}(609.79 \AA)$ \\
Slit dimensions $(x \times y)$ & $2^{\prime \prime} \times 240^{\prime \prime}$ \\
Spatial resolution along $y$ & $1.68^{\prime \prime}$ \\
Average temporal resolution $\Delta t$ & $24.6 \mathrm{~s}$ \\
Duration & $7 \mathrm{~h} 10$ \\
Beginning (UT) & $6: 40$ \\
End (UT) & $13: 51$ \\
\hline
\end{tabular}

In both cases, however, wavelet analysis of the observed signals in the two filaments leads to evidence of intermediateperiod, small-amplitude oscillations (less than $20 \mathrm{~km} \mathrm{~s}^{-1}$ ) apparently simultaneously with a total or partial eruption of the filaments. Moreover, the CDS observations in He I at $584.33 \AA$ and in $\mathrm{Mg} \mathrm{X}$ at $609.79 \AA$ reveal intensity oscillations in phase in both lines, preceded by velocity oscillations in phase in both lines. Both data sets reveal a vertical oscillatory motion inside the filaments: the CDS observations in He I at $584.33 \AA$ exhibit a damped oscillation. The DST 2D imaging observations in the $\mathrm{H} \alpha$ wings reveal that parts of the filament are moving vertically in opposite directions, while the filament is moving over the solar surface, before its disappearance. No damping was measured for this event. In addition, a flare and a loop eruption are observed in Yohkoh/SXT.

\section{Damped oscillatory motions in an eruptive filament, observed simultaneously in $\mathrm{He} \mathrm{I}$ and $\mathrm{Mg} \mathrm{X}$}

\subsection{CDS data set properties}

During the 11th MEDOC campaign we obtained long observations of a filament with CDS. The observations were carried out on May 30, 2003, between 6:40 UT and 13:51 UT, in He I at $584.33 \AA$ and $\mathrm{Mg}$ X at $609.79 \AA$. Table 1 summarizes the observational parameters of the sequence.

At the beginning of the observations, the slit centre was located on the solar disc at $x=690$ arcsec and $y=418$ arcsec. During the observations, the slit's $x$ position was regularly shifted to compensate for solar rotation in order to follow the same part of the filament. At the end of the observation the slit was at $x=721$ arcsec. The data have three dimensions: time, wavelength, and spatial position on the solar disc. At each time $t_{i}$, a spectrum within a constant wavelength range $\Delta \lambda$ (centred on either the He I or Mg X line) was obtained for each $y_{j}$ position along the slit, which is 141 pixels high ( 240 arcsec). The usual corrections were applied to these data: flat fielding, cosmic ray removal, and correction of some geometric instrumental effects. The method applied to identifying the signal due to the filament is described by Pouget et al. (2006): for each time $t$, the pixels $(t, y)$ that belong to the filament were those whose intensity is below a threshold chosen such that the filament width remained constant in time.

To compute the Doppler velocity, we used a reference profile, averaged over the whole field of view and over time: the velocity studied below is consequently a relative quantity, but the spectral analysis is not affected. For each pixel, the line profile is fitted to a Gaussian profile, plus a component including the asymmetric shape of the profiles obtained by CDS after the 1998 SOHO recovery (Thompson 1999), allowing the extraction 


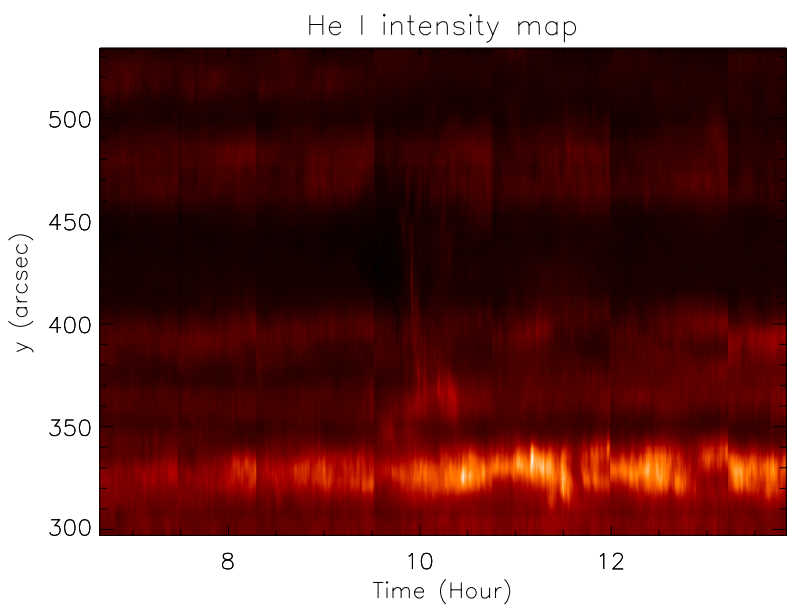

Fig. 1. He I intensity map, as a function of time and along the slit. The filament is located between $y=409 \operatorname{arcsec}$ and $y=459 \operatorname{arcsec}$.

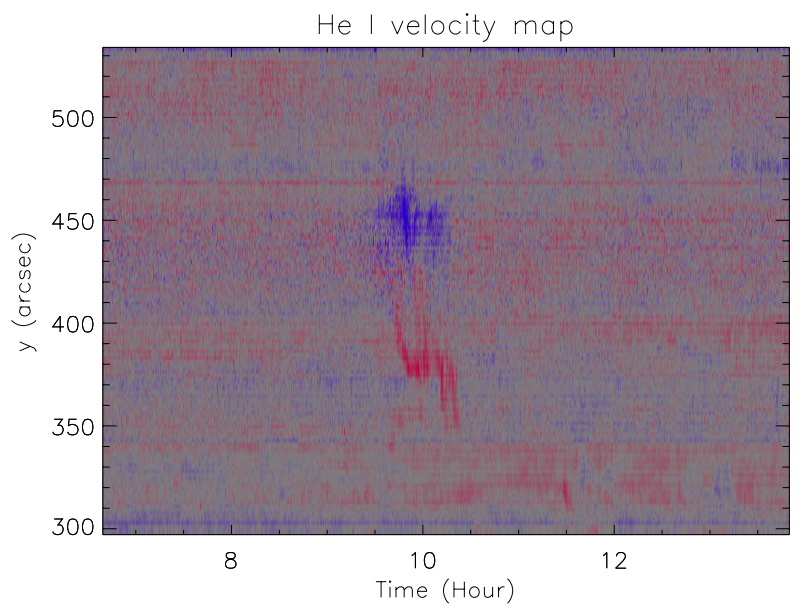

Fig. 2. He I velocity map, as a function of time and along the slit. The filament is located between $y=409$ and 459 arcsec.

of its central wavelength ${ }^{1}$. However, it should be noted that it is very difficult to get accurate absolute lineshifts measured by CDS (Pouget et al. 2006). The typical uncertainty of the relative velocities is $6 \mathrm{~km} \mathrm{~s}^{-1}$, owing to the uncertainty of the pixel position on the CCD in the spectral dimension.

Figures 1 and 2 show the intensity averaged over the waveband centred on He I and the associated velocity series: the filament is visible in intensity as the dark zone between slit positions $y=409$ arcsec and 459 arcsec. The eruption occurred between 9:00 and 11:00 UT, and a strong blue shift is visible in the filament, while a strong red shift is visible between the positions $y=350 \operatorname{arcsec}$ and $y=400 \operatorname{arcsec}$, below the position of the filament (Fig. 2). At the same time, an intensity enhancement around position $y=334$ arcsec is clear (Fig. 1).

Figures 3 and 4 show the He I and Mg X profiles respectively, averaged over the $y$ dimension and the whole temporal sequence, which are then compared to the $\mathrm{He} \mathrm{I}$ and $\mathrm{Mg} \mathrm{X}$ profiles, averaged over the filament, between the positions $y=409$ arcsec and $y=459 \operatorname{arcsec}$ (and the whole temporal sequence). The profiles are in emission, but the filament profile is clearly less intense by more than a factor of two at its maximum than the average profile along the slit, for both lines. The optically thick He I line is

\footnotetext{
1 The routine used is FIT_SPEC, available in the SolarSoftWare Library of IDL.
}

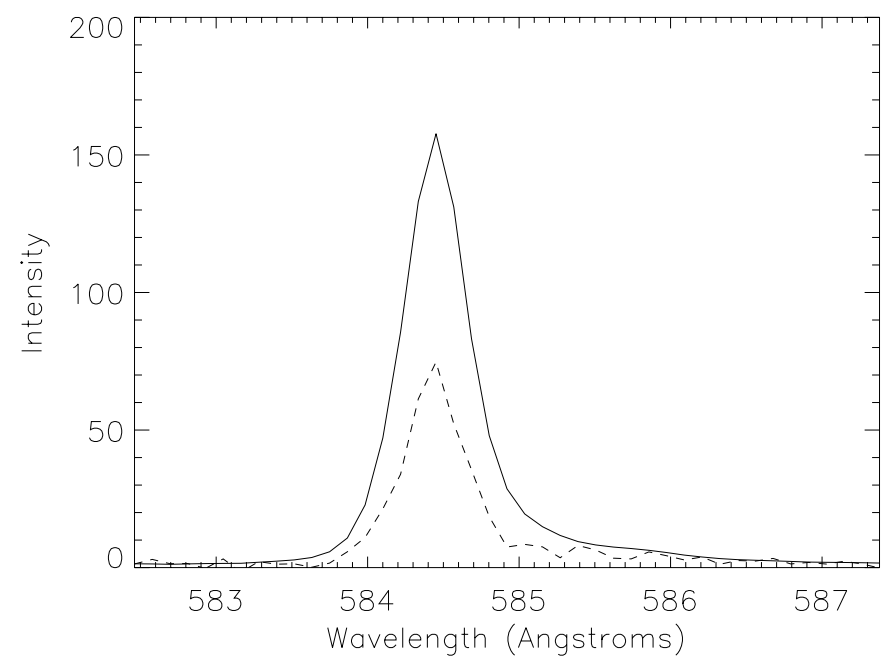

Fig. 3. Mean He I profiles (arbitrary units): solid line, average over the $y$ dimension and over the whole temporal sequence; dashed line, average over the filament and the whole temporal sequence.

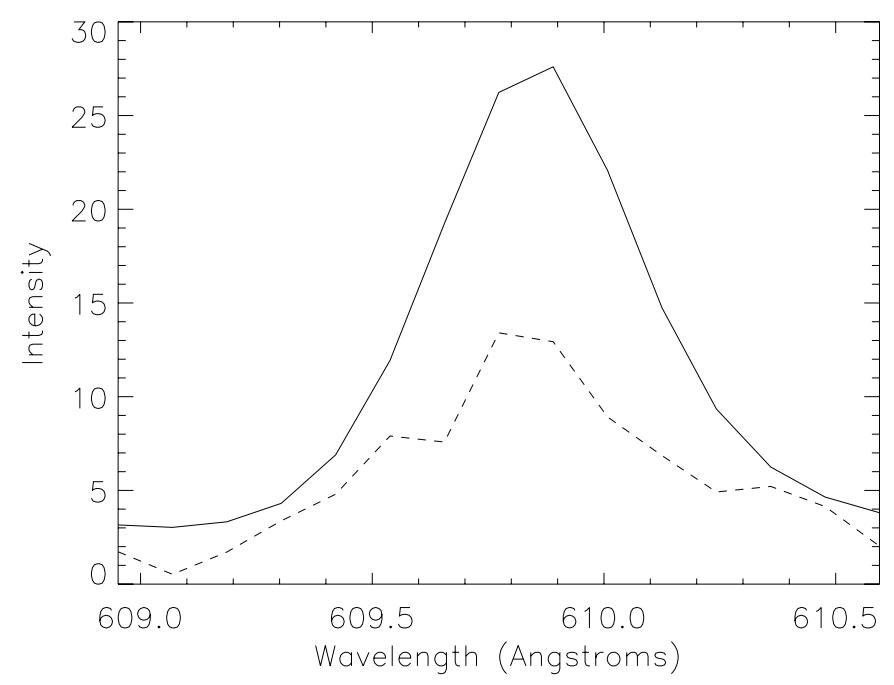

Fig. 4. Mean $\mathrm{Mg} X$ profiles (arbitrary units): solid line, average over the $y$ dimension and over the whole temporal sequence; dashed line, average over the filament and the whole temporal sequence.

formed at a temperature $T \sim 20000 \mathrm{~K}$, as mentioned by Régnier et al. (2001), and it is sensitive to the motions of the prominence, while the coronal $\mathrm{Mg} \mathrm{X}$ line is formed at $T \sim 1.2 \mathrm{MK}$.

\subsection{Overview of the observed area}

The EIT (Delaboudinière et al. 1995) instrument provided the contextual field of view. Figure 5 shows (top left) an EIT image of the area around the filament studied in He II at $304 \AA$ obtained at 9:35 UT. The CDS slit position is indicated by a vertical black line on the disc. Figure 6 shows an $\mathrm{H} \alpha$ image obtained at 8:07 UT at the Kiepenheuer Institute.

The EIT differences between two successive images in $304 \AA$ (Fig. 5) reveal that a part of the filament lifts off from 9:35 to 10:35 UT (simultaneously with the CDS observations) and is seen as a prominence on the west limb, while the part on the disc does not erupt. The prominence disappears into the corona but is not associated with a visible coronal mass ejection, as confirmed by SOHO/LASCO observations. The event is comparable 

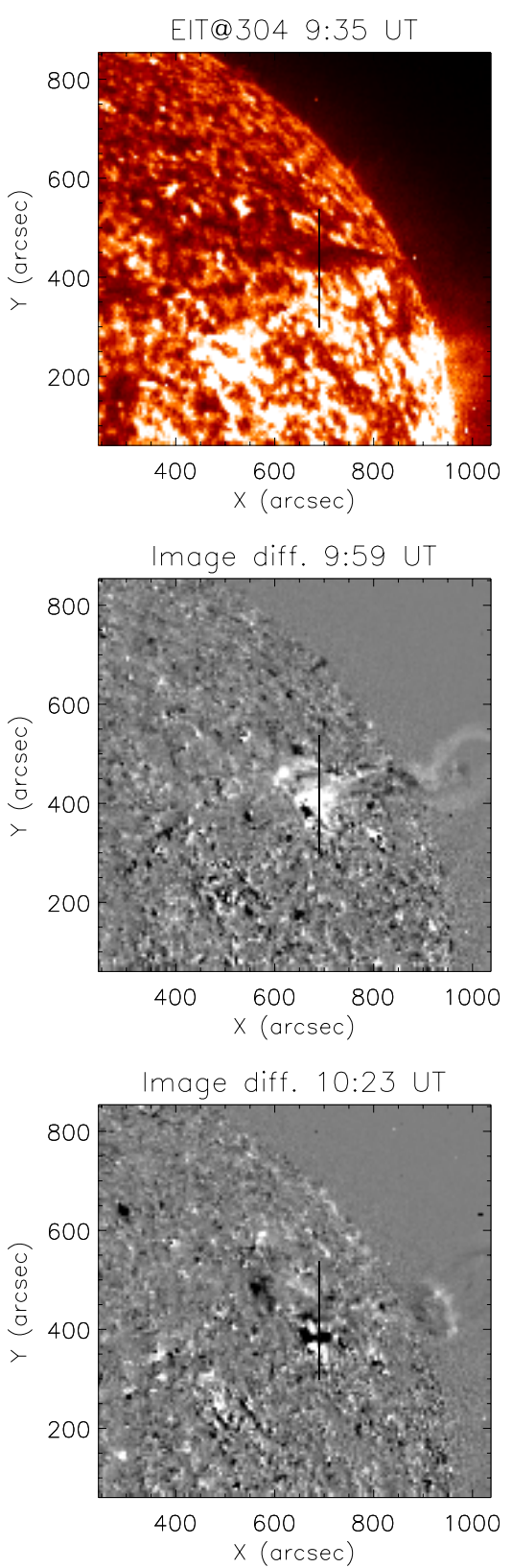
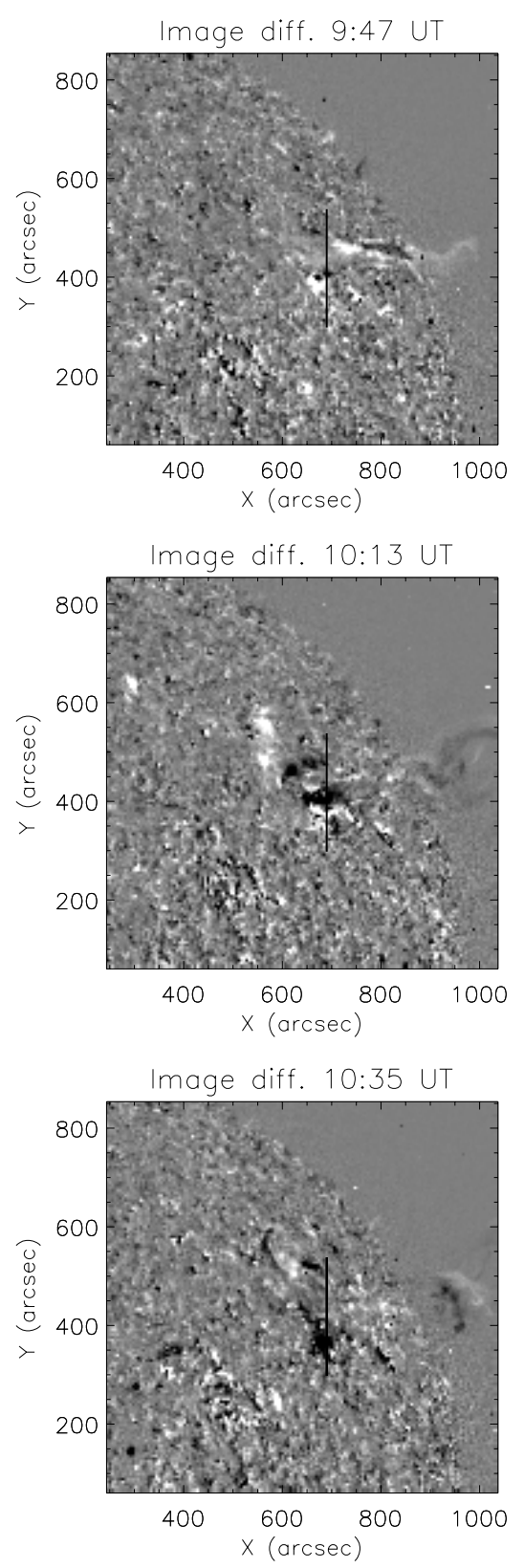

Fig. 5. Top left: EIT image of the area of the filament studied, in $304 \AA$ A obtained at 9:35 UT, on May 30, 2003. Top right, middle, and bottom rows: EIT difference image sequence of the filament in $304 \AA$ between 9:47 and 10:35 UT (from left to right, top to bottom), obtained at a nearly 12-min cadence. The filament eruption begins at 9:35 UT. The CDS slit position is indicated by a vertical black line. to the so-called confined filament eruption studied by Filippov \& Koutchmy (2002).

The CDS slit cuts across the part of the filament that does not erupt. The velocities detected in this part of the filament could be the signature of an excitation due to the erupting part of the filament. Previously, a flare was observed with EIT in the same region at 6:30 UT. The source of the destabilization of the filament could be a wave resulting from this flare. The sequence of MDI magnetograms (Scherrer et al. 1995) obtained between 8:03 UT and 12:47 UT does not exhibit any particular signature that could be linked to the eruption.

\subsection{Spectral analysis of the signal in the filament}

The Doppler velocity and the intensity time series are derived in the filament for both lines, $\mathrm{He} \mathrm{I}$ and $\mathrm{Mg} \mathrm{X}$, by averaging the 30 profiles between the positions $y=409 \operatorname{arcsec}$ and 459 arcsec, for each temporal exposure. In the following, we used a wavelet analysis to explore their frequency content because the signals show strong variations in time.
Results in intensity: Fig. 7 shows the intensity variations and the corresponding wavelet analysis for the $\mathrm{He} \mathrm{I}$ and $\mathrm{Mg} \mathrm{X}$ lines. We used the code written by Torrence \& Compo (1998) ${ }^{2}$. In both lines, the intensity begins to decrease slowly before the eruption, and then increases impulsively at the beginning of the eruption, around 9:55 UT, simultaneously in $\mathrm{He} \mathrm{I}$ and $\mathrm{Mg}$ X. The intensity in He I oscillates with a period around $22 \mathrm{~min}$ from 9:00 UT to 11:00 UT. The intensity oscillates also with a period around 80 min from 9:00 UT to 13:00 UT. In Mg X, the intensity oscillates with a period around $30 \mathrm{~min}$, from 9:00 UT to 11:00 UT and also oscillates with a period around $80 \mathrm{~min}$ from 9:00 UT to 11:00 UT.

Results in velocity: Fig. 8 shows the velocity and the corresponding wavelet analysis in $\mathrm{He} \mathrm{I}$ and $\mathrm{Mg} \mathrm{X}$. In both lines, a strong upward impulse is detected simultaneously with the eruption around 10:00 UT. The wavelet analysis confirms that the

${ }^{2}$ Available at http://paos. colorado.edu/research/wavelets 


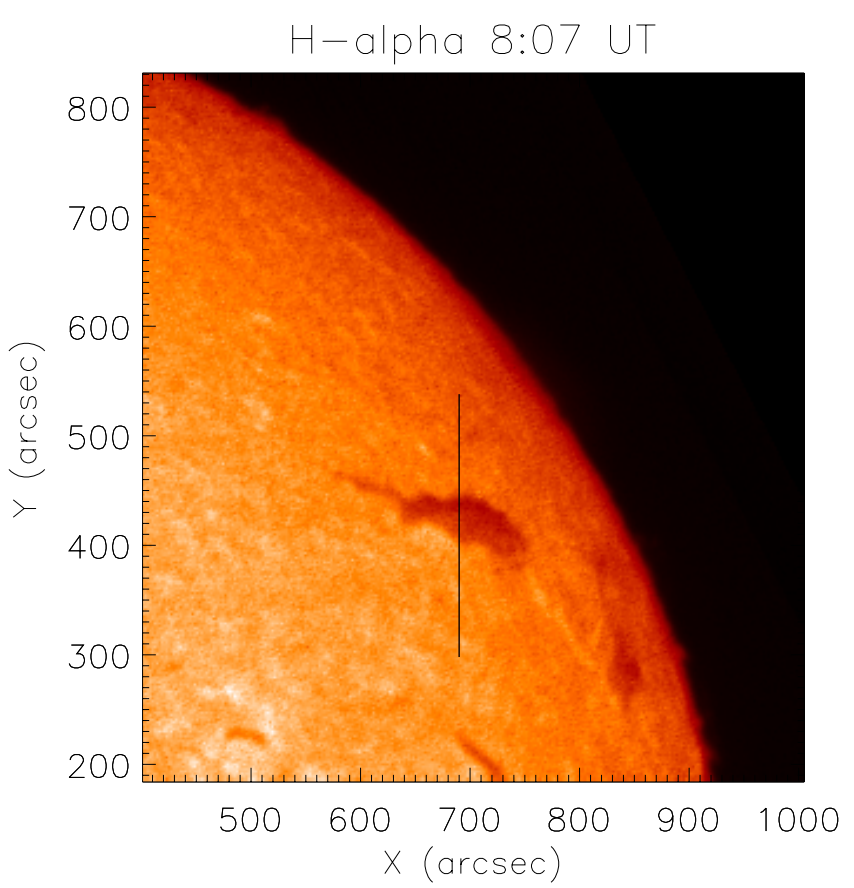

Fig. 6. Kiepenheuer Institute image of the area of the filament studied, in $\mathrm{H} \alpha$, obtained at 8:07 UT on May 30, 2003. The CDS slit position is indicated by a vertical black line.

period is about $20 \mathrm{~min}$ in both lines. $\mathrm{Mg} \mathrm{X}$ also exhibits tenminute period oscillations. The oscillations last roughly one hour and are quickly damped.

Figure 9 displays a close-up around the eruption of the intensity and velocity in both lines simultaneously (in arbitrary units, for easier temporal comparison between the signals). The velocity oscillations in $\mathrm{Mg} \mathrm{X}$ are in phase with the velocity oscillations in He I, suggesting that the hot corona in which the filament channel is embedded responds coherently to the oscillating filament. The intensity signals in both lines are in phase, showing simultaneous variations as well. Moreover, the peak of intensity seen in both lines appears after the beginning of the oscillations detected in velocity, which indicates that the dynamical effects precede the thermal effects.

A more refined analysis of the velocity shows that the signal in He I can be separated into two parts: one related to the oscillation itself with an apparent period around 22 min seen with the wavelet analysis in Fig. 8 and another with a period longer than $80 \mathrm{~min}$. The residual velocity (after the subtraction of the long-period trend using a $50 \mathrm{~min}$ boxcar running average) is displayed in Fig. 10 and corresponds to an oscillation that can be fitted by an exponentially damped sine function, with a frequency linearly decreasing with time. The velocity can then be expressed as: $v(t)=A \sin \left(2 \pi\left(b-a\left(t-t_{0}\right)\right)\left(t-t_{0}\right)+\phi\right) e^{-\left(t-t_{0}\right) / \tau}$, with $A=12.2 \mathrm{~km} \mathrm{~s}^{-1}, a=2.3410^{-4} \mathrm{mHz} / \mathrm{s}, b=1.26 \mathrm{mHz}$, $\phi=3.02 \mathrm{rad}, \tau=25.08 \mathrm{~min}$ and $t_{0}=9.78 \mathrm{~h}$, with $\tau / T=1.9$ (and $T=1 / b$ the initial period of the oscillations). The pseudoperiod becomes roughly $50 \mathrm{~min}$, one hour after the velocity maximum.

\subsection{Summary of the analysis}

The analysis of the CDS observations performed on an eruptive filament in the $\mathrm{He} \mathrm{I}$ and $\mathrm{Mg} \mathrm{X}$ lines exhibits strong intensity and velocity oscillations during the eruption. Periods range from 20 to $30 \mathrm{~min}$ in intensity, periods longer than $80 \mathrm{~min}$ are also detected. For the velocity measured in He I, the amplitude of the main 20-min period oscillations is $12.2 \mathrm{~km} \mathrm{~s}^{-1}$, with a damping time of $25 \mathrm{~min}$. The EIT $304 \AA$ images show that the filament erupts during the CDS observations.

Figure 9 compares the signals in intensity and velocity. First, one can note that the oscillation in velocity starts at the same moment as a long-period signal in intensity but that a sudden brightening in intensity occurs well after the oscillations have started. This could suggest that a thermal event occurs after the oscillatory behaviour started. More precisely, just before the brightening, between 9.83 UT and 9.95 UT, one can distinguish shortperiod oscillations of a few minutes in the He I velocity signal. These oscillations can be seen on the wavelet diagrams Figs. 7 and 8 , both in intensity and velocity, in He I.

In the next section, we analyse the similar period oscillations detected in another eruptive filament, measured from the ground, in the wings of the $\mathrm{H} \alpha$ line.

\section{Oscillation and rotational motions detected in an eruptive filament, observed in the wings of $\mathrm{H} \alpha$}

\subsection{DST dataset properties}

In the following, we analyse ground-based observations of a similar event observed in NOAA AR 7779 on September 18, 1994, in the blue and red wings of $\mathrm{H} \alpha$ line at $\lambda_{ \pm}=6562.8 \pm 0.75 \AA$, from 15:32 UT to 17:22 UT, using a universal birefringent filter $(\mathrm{UBF})$, operated in the $0.24 \AA$ full width at half maximum $(F W H M)$ mode. This event occurred very near the solar disc centre, while the event being studied previously was located near the limb. The square images are 350 arcsec wide and centred on $\mathrm{N}$ 17.3, E 1.3. Each set of filtergrams was obtained at a cadence of one minute, with a 0.34 arcsec/pixel spatial resolution, during 110 min at the NSO/Sacramento Peak Dunn Solar Telescope (Baudin et al. 1997, 1998).

The photospheric context of the region, including a discussion of dynamical effects, was given in a preliminary paper by Baudin et al. (1997). Following this observation sequence, a large soft X-ray (SXR) eruption was recorded by the Yohkoh Soft X-Ray Telescope (YOHKOH/SXT) (Fig. 11).

Abramenko et al. (1996) have obtained vector magnetograms of NOAA AR 7779 and determined the helicity and corresponding electric currents. Although AR 7779 was located close to the equator (N17E02), the negative current helicity found is typical of a northern hemisphere event, and the value of the current helicity could satisfy the energy requirements for the filament eruption.

Our analysis is now focused on the study in intensity and Doppler velocity of the eruption of the filament centred in the f.o.v. The wing intensity is the sum of the signals obtained in the red and blue wings. The Doppler velocity is obtained using the following method (Malherbe et al. 1997; Georgakilas et al. 2001): an $\mathrm{H} \alpha$ reference centre-disc profile (Delbouille et al. 1973) ${ }^{3}$ is convolved with a Gaussian profile with a $0.24 \AA F W H M$, corresponding to the width of the filter used for the observations. This profile is shifted by several steps $\Delta \lambda=\frac{F W H M}{2}$. For each shifted profile, the ratio $\frac{I_{2}-I_{1}}{I_{2}+I_{1}}$ is computed, where $I_{2}$ is the intensity in the red wing and $I_{1}$ the intensity in the blue wing at $\lambda_{ \pm}=6562.8 \pm 0.75 \AA$. A calibration

\footnotetext{
${ }^{3}$ Available at the BASS2000 database: http://bass2000.bagn. obs-mip.fr/
} 

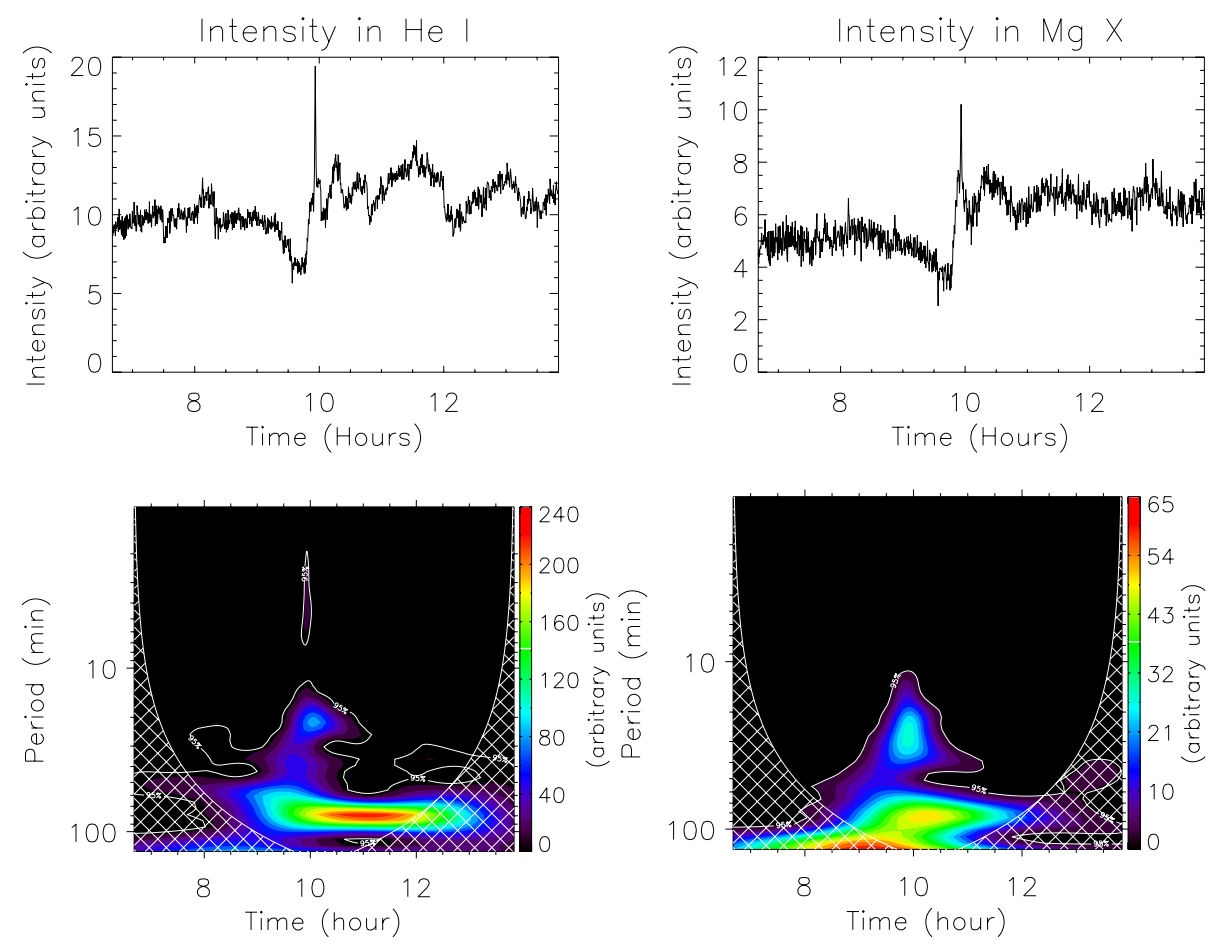

Fig. 7. Intensity in the filament (top) as a function of time in He I (left) and in Mg X (right), and the corresponding wavelet analysis (bottom); significant power is within the $95 \%$ confidence level contour and outside the hatched cones of influence.
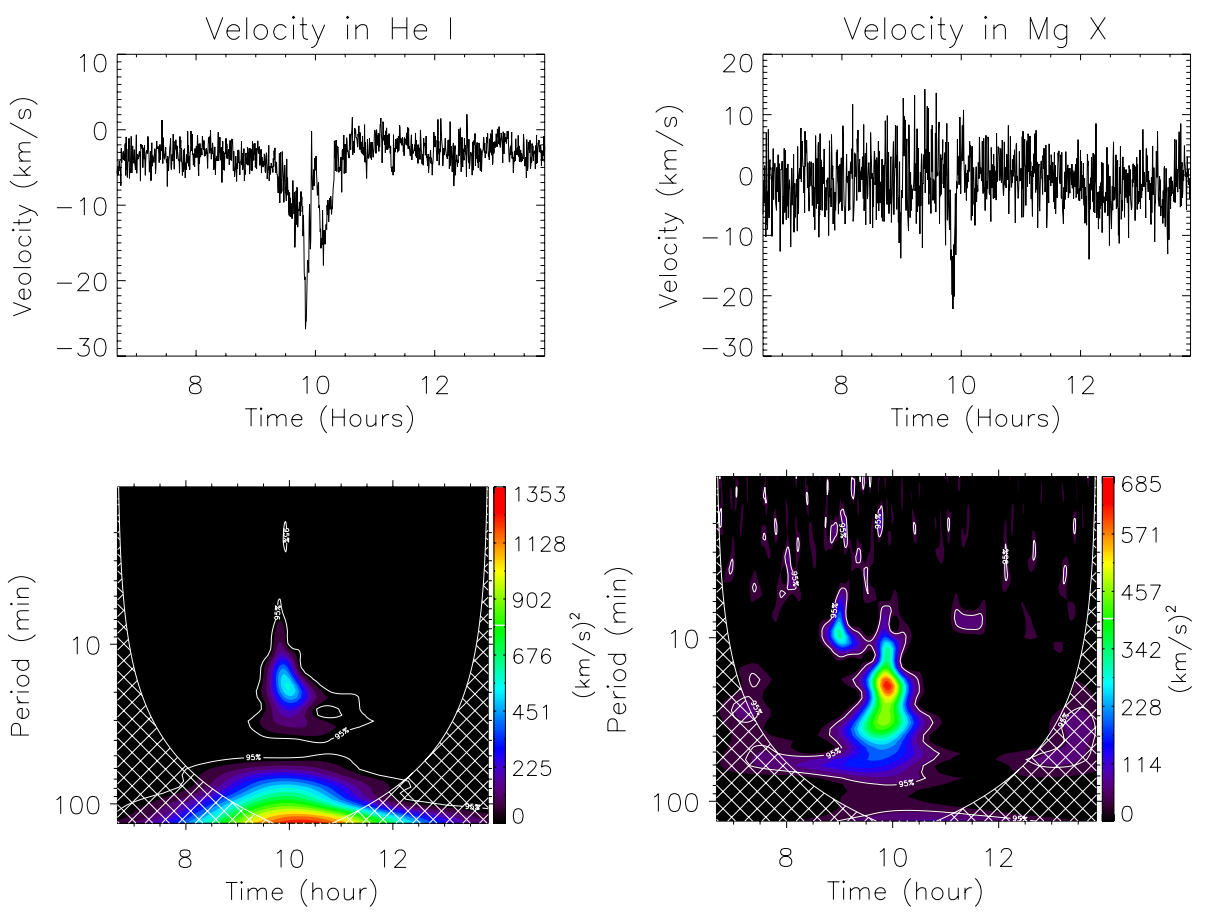

Fig. 8. Velocity in the filament (top), as a function of time, in He I (left) and in Mg X (right), and the corresponding wavelet analysis (bottom); significant power is within the $95 \%$ confidence level contour and outside the hatched cones of influence.

curve $\frac{I_{2}-I_{1}}{I_{2}+I_{1}}=f(v)$ is obtained by assuming that the shifting is equal to $\frac{v}{c} \lambda_{0}$, with $\lambda_{0}=6562.8 \AA$. The curve is displayed in Fig. 12, assuming no differential changes of the line profile during the event. We note that this assumption is modelindependent but may lead to an underestimation of the deduced velocities (Labrosse et al. 2010). The velocities are readily deduced from this calibration curve, in agreement with the calibration of Georgakilas et al. (2001) who used the same UBF mode.

Figures 13 and 14 show the $\mathrm{H} \alpha$ summed wings intensity sequence, and Figs. 15 and 16 show the velocity sequence, from $t=38$ to $t=72 \mathrm{~min}$ after the beginning of the observations. The eruption occurred beween $t=38$ and $50 \mathrm{~min}$. A large part of the filament erupted as the filament moves towards the northwest, with an apparent proper motion of up to $25 \mathrm{~km} \mathrm{~s}^{-1}$, as measured between $t=42 \mathrm{~min}$ and $t=54 \mathrm{~min}$ (Baudin et al. 1998). The velocity sequence shows some vertical motions, as the two parallel blue and red areas clearly reveal: parts of the filament are moving upward (blue areas), whilst parts of the filament are moving downwards (red areas). The velocity ranges from 6 to $20 \mathrm{~km} \mathrm{~s}^{-1}$ for the zone going downwards and from 5 to $15 \mathrm{~km} \mathrm{~s}^{-1}$ for the ascending zone. A possible interpretation of the sequence is to assume rotationally coherent motions during approximately ten minutes (from $t=40$ to $t=50 \mathrm{~min}$ ), in order to satisfy the 


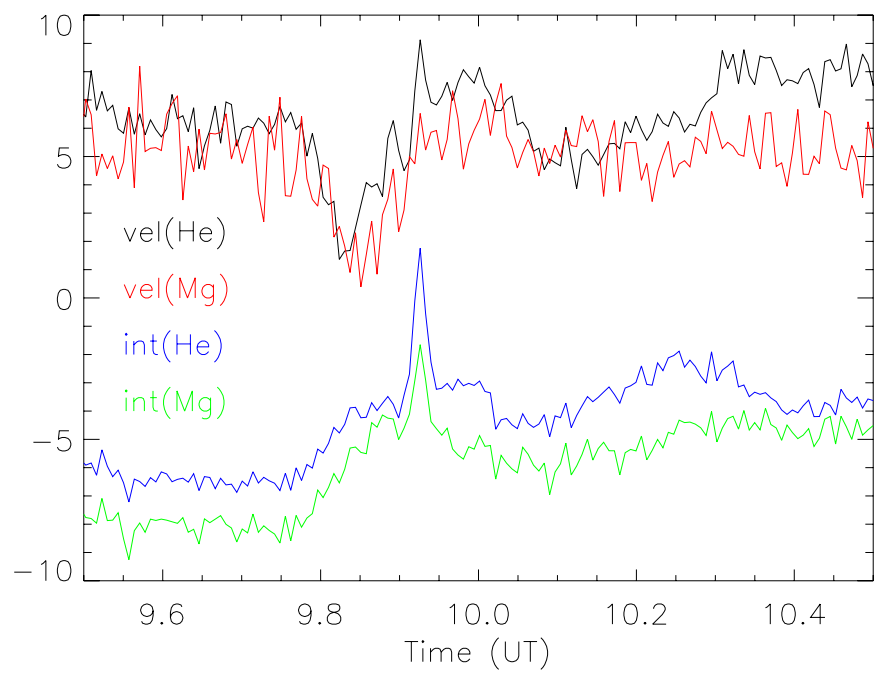

Fig. 9. Comparison of the signals in the filament between 9:00 UT and 11:00 UT, in intensity (He I in blue and $\mathrm{Mg} \mathrm{X}$ in green) and in velocity (He I in black and $\mathrm{Mg} \mathrm{X}$ in red).

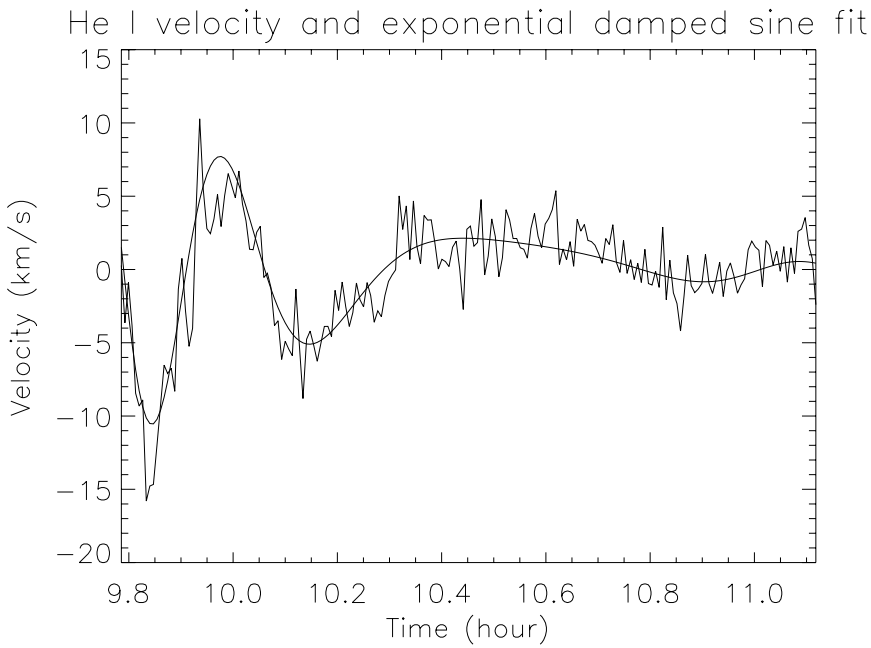

Fig. 10. Residual velocity in the filament in He I during the eruption, and its fit, with the lond period signal removed to emphasize and fit the low period signal.

mass conservation condition assuming no significant change in ionization ratio (temperature) during this interval (see Sect. 4). At the end of the eruption, a part of the filament fell down (only the red part of the velocity remained, Fig. 16) while the signature of the filament in intensity was less extended (Fig. 14) because the filament lost a part of its mass during the eruption (disappearance). From $t=70 \mathrm{~min}$, the signature of a new filament is clear in intensity and velocity along the filament channel.

Two hours after the eruption, a large-scale loop system was observed by Yohkoh in SXR moving outward, suggesting that strong effects were produced in the hot surrounding corona.

\subsection{Spectral analysis of the signal in the filament}

Power spectral maps are computed from the velocity signal, using the fast Fourier transform. The power maps lead us to retain four spatial zones with high power, over several consecutive maps. These zones are indicated by the numbers 1-4 in Figs. 13 and 16. The zones correspond to the different positions of the filament whilst it is moving toward the northwest: for zone 1 between $t=38$ and $t=42 \mathrm{~min}$ after the beginning of the observations, for zone 2 between $t=44$ and $t=48 \mathrm{~min}$, for zone 3 , at $t=50$ and $52 \mathrm{~min}$, and for zone 4 , at $t=54 \mathrm{~min}$. Figure 17 shows the four power maps averaged over the intervals [15, $55 \mathrm{~min}$ ], [8, $15 \mathrm{~min}]$, [4, $8 \mathrm{~min}]$, and [2, $4 \mathrm{~min}]$, corresponding to the intervals observed on the filament. Zone 1 is visible in the range [4, $8 \mathrm{~min}$ ] and in the range [2, $4 \mathrm{~min}$ ] (bottom left and right). Zone 2 is visible in the range [8,15 min] (top right). Zone 3 is visible in the range $[15,55 \mathrm{~min}]$ (top left). Zone 4 is visible in the range [4, 8 min] (bottom left).

The wavelet analysis of the Doppler velocity for these four zones using the full $110 \mathrm{~min}$ long sequence of the observations is displayed in Fig. 18:

- in zone 1, the period ranges from three to ten minutes, as already shown in Fig. 17 at the beginning of the sequence;

- zone 2 has a period of about $12 \mathrm{~min}$ and a period of $45 \mathrm{~min}$ centred on $t=55 \mathrm{~min}$, during the rotational motion;

- zone 3 has a weak period ranging from 8 to 40 min centred on $t=60 \mathrm{~min}$;

- zone 4 shows significant power near 7 and 20 min periods centred on $t=65$, while the filament was falling down.

\subsection{Summary of the analysis}

A 2D analysis of $\mathrm{H} \alpha$ intensity observations in a filament during 110 min reveals a horizontal displacement of the filament at a speed of $25 \mathrm{~km} \mathrm{~s}^{-1}$ during $12 \mathrm{~min}$, along with a vertical motion deduced from the Doppler velocity signal, followed by the eruption of a large part of the filament and the downward motion of the rest of the filament. During ten minutes, rotational motions inside the filament are detected before the occurence of the fast proper motion, starting at $t=40 \mathrm{~min}$ towards the northwest. Wavelet analysis of the velocity shows four zones with high power and different behaviours. The Doppler velocity in these zones exhibits strong variations (from a few $\mathrm{km} \mathrm{s}^{-1}$ to more than $10 \mathrm{~km} \mathrm{~s}^{-1}$ ) and periods ranging from three to ten minutes. Periods around $20 \mathrm{~min}$ and $45 \mathrm{~min}$ are also measured.

\section{Conclusion and discussion}

We presented the results of the analysis of two eruptive filaments exhibiting some similarities observed in completely different manners: one observed in $\mathrm{He} \mathrm{I}$ and $\mathrm{Mg} \mathrm{X}$ with a space-borne spectrometer, the other observed in $\mathrm{H} \alpha$ wings with a groundbased imager. In both cases, the eruptive filaments show small amplitude oscillatory motions associated with an eruption and a partial disappearance: the filaments lost a part of their mass because they are heated and evaporate.

In $\mathrm{He} \mathrm{I}$, the pseudo-period of the velocity oscillations is increasing from 20 to $50 \mathrm{~min}$, whilst the amplitude is decreasing. This damped velocity oscillation could be interpreted, as suggested by Vrsnak et al. (1990), as the signature of the eruption, owing to the weakening of the restoring force, giving rise to the increasing period. In this model, when dealing with the oscillatory motions in an eruptive filament, the authors explain that once the filament is in its eruptive phase, the filament jumps from an unstable position to a stable one at a higher altitude. In this stable position, if the damping factor (inverse of the damping time) is less than the frequency of the first oscillation mode, the filament will oscillate around its equilibrium position, as a damped oscillator. This behaviour corresponds to what is described by our observations in Sect. 2. An increased heating 

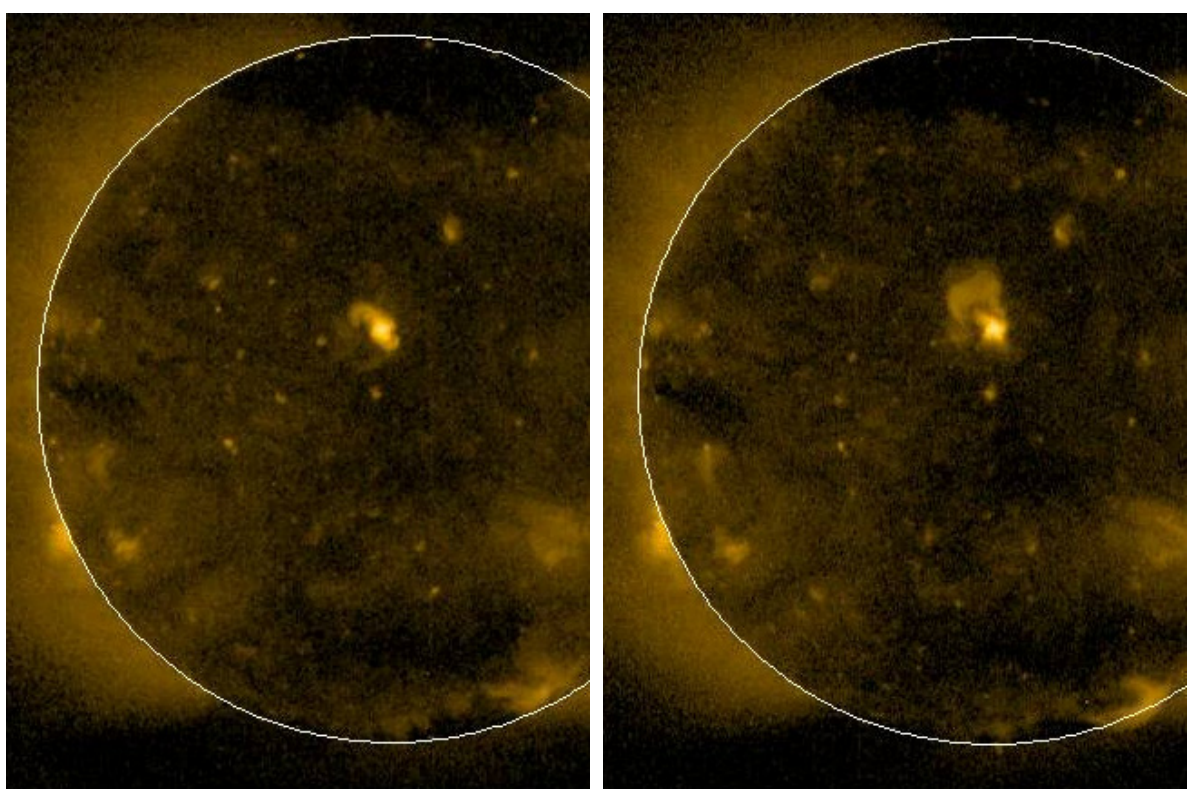

Fig. 11. Yohkoh/SXT images taken at 15:32 UT (left) and 19:18 UT (right) on September 18, 1994, showing the observed region and its eruption, near the disc centre.

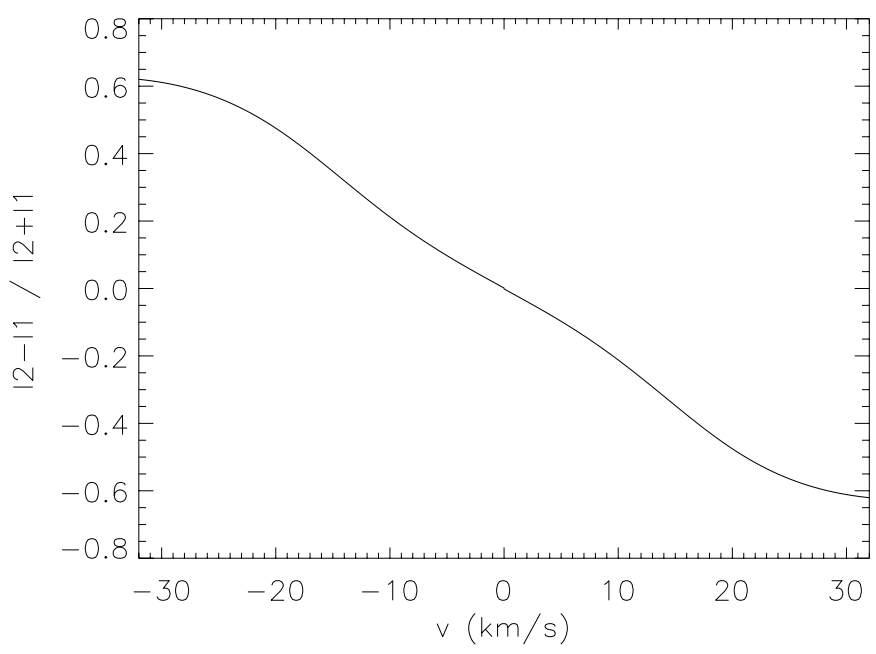

Fig. 12. Calibration curve $\frac{I_{2}-I_{1}}{I_{2}+I_{1}}=f(v)$ for NSP/DST observations.

of the top part of the filament is another possibility suggested by Filippov \& Koutchmy (2002).

We can link this increase in the period of the oscillating filament to the decreasing stiffness of an oscillating spring. This suggests the weakening of the restoring force, which may also be related to the destabilization and onset of the filament eruption that has modified the whole structure of the filament, hence the restoring forces (Isobe \& Tripathi 2006).

The value of $\tau / T=1.9$ obtained in our study may be compared to the one obtained for similar behaviour in a longitudinally damped oscillation of a filament observed in $\mathrm{H} \alpha$ by Vršnak et al. (2007): $\tau / T=2.3$. They interpret their observations in terms of poloidal magnetic flux injection by magnetic reconnection at one of the filament legs. They also point out an increase with time in the oscillation period and interpret this behaviour in terms of nonlinear effects in the framework of a harmonic oscillator model.

Results based on the velocity in $\mathrm{He} \mathrm{I}$ and $\mathrm{Mg} \mathrm{X}$ indicate that the corona in which the filament channel is embedded responds coherently with the oscillating filament. The intensity signals in both lines are very close in phase, showing nearly simultaneous variations. Moreover, the peak of intensity seen in both lines appears after the beginning of the oscillations detected in velocity, which indicates that the dynamical effects precede the thermal effects.

For the second case, the analysis of the velocity in $\mathrm{H} \alpha$ shows a strong power peak detected for zone 1 in the extreme eastern part of the filament rooted in the chromosphere: the low values of the period are close to the typical periods of the photospheric (5-min) and chromospheric (3-min) oscillations. These oscillations are detected before the motion of the filament toward the northwest and before its eruption. We suggest that the filament is excited by the photospheric and chromospheric oscillations (Bocchialini et al. 2001), which are at the origin of the motion of the filament, with a triggering of the eruption and the flare due to reconnections. This result can be compared to the four-minute periods obtained in $\mathrm{H} \alpha$ (Schmieder et al. 1991) in chromospheric filamentary structures, interpreted either in terms of eigenmodes excited by the chromospheric oscillations or in terms of Alfvèn waves generated by the chromosphere and transmitted to the filamentary structures, as confirmed by the Hinode/SOT observations (Okamoto et al. 2007).

Indeed, these proper motions are difficult to interpret as definite horizontal velocities. It seems rather that we are dealing with increasing turbulent proper motions with increasing ionization ratio (temperature) leading to the disappearance of a large part of the filament after $t=54 \mathrm{~min}$. The analysis of the velocity, for both datasets, revealed the presence of oscillations with a period from 20 to 30 min in $\mathrm{He} \mathrm{I,} \mathrm{Mg} \mathrm{X}$, and $\mathrm{H} \alpha$, simultaneously with the eruption of the filaments. These periods can be compared to the 20-min period found by Anzer (2009), who discusses the different oscillation periods for global modes of quiescent prominences. Anzer uses simple 1D prominence configurations to describe the magnetohydrostatic equilibrium and their small amplitude oscillations. The restoring force along the $z$-direction, perpendicular to the solar surface, resulting from the magnetic tension of the stretched field lines, leads to periods of $20 \mathrm{~min}$ in this direction. Our results can also be compared to those of Terradas et al. (2005), who show that, for a typical prominence temperature of $6000 \mathrm{~K}$, the oscillation period computed for a quiescent prominence is around $25 \mathrm{~min}$ and the damping time is around $19 \mathrm{~min}$. The damping time increases with temperature 
K. Bocchialini et al.: Oscillatory motions observed in eruptive filaments
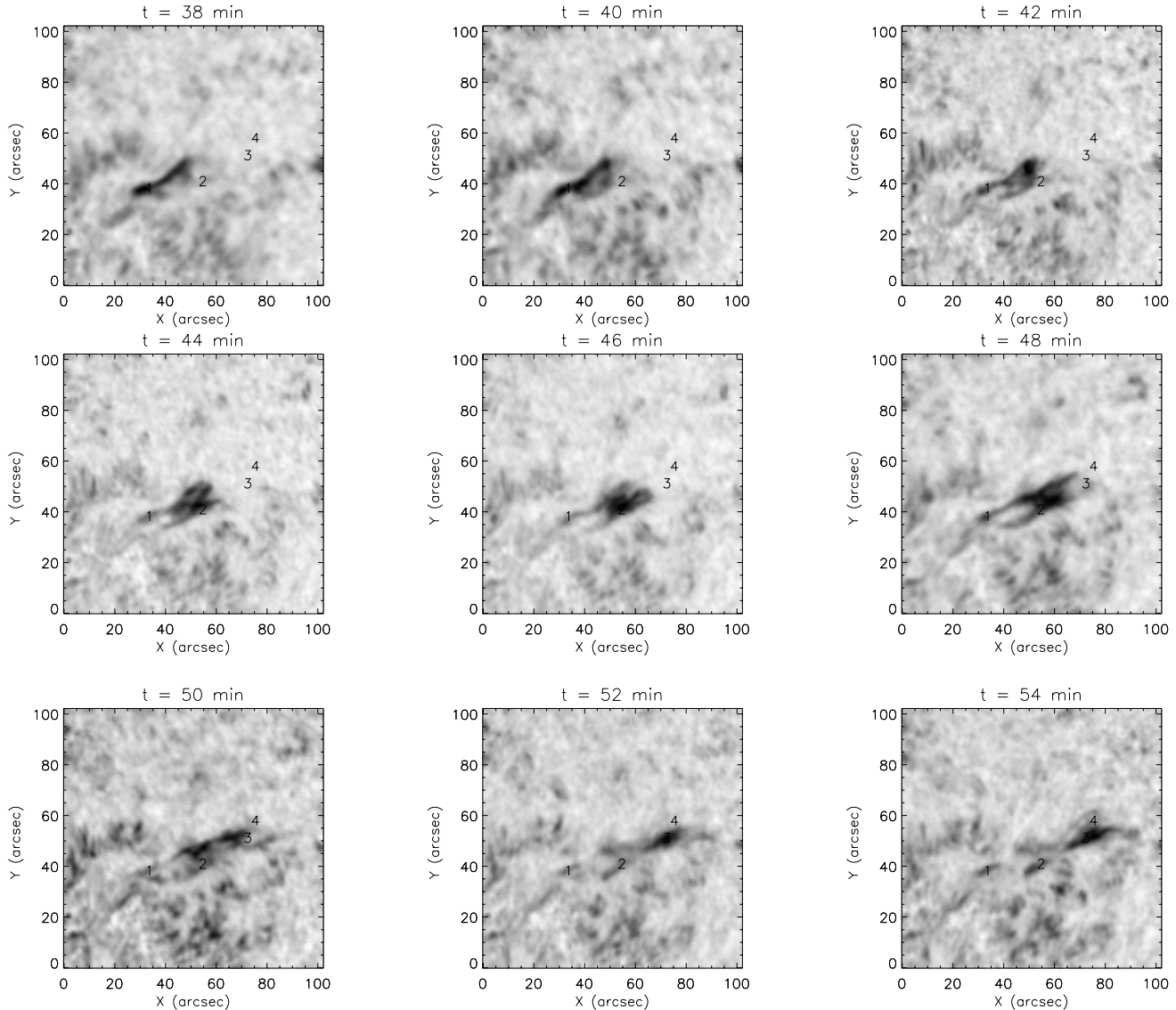

Fig. 13. Part of the intensity sequence in summed $\mathrm{H} \alpha$ wings from $t=38 \mathrm{~min}$ to $t=54 \mathrm{~min}$, after the beginning of the observations.
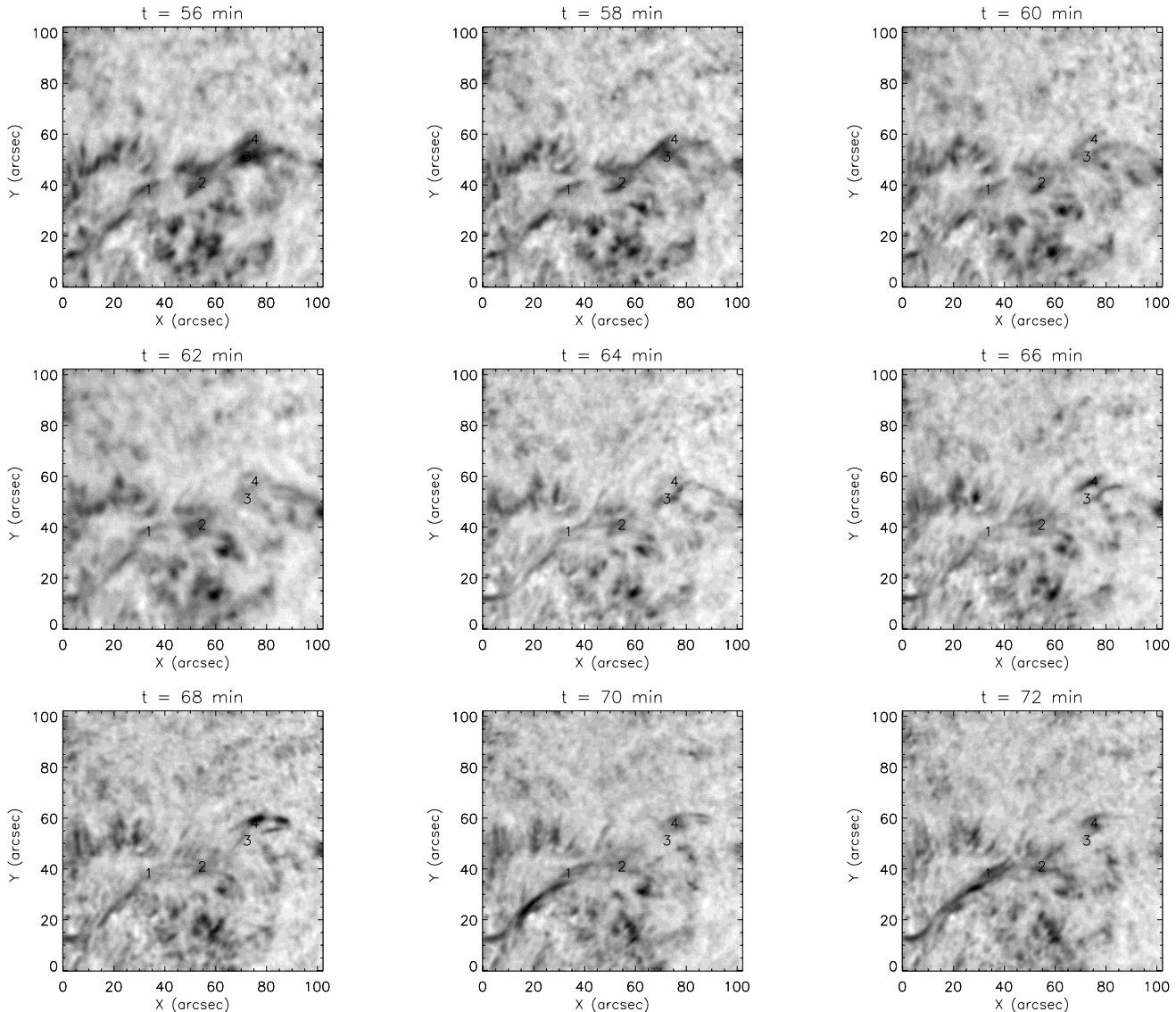

Fig. 14. Part of the intensity sequence in summed $\mathrm{H} \alpha$ wings from $t=56 \mathrm{~min}$ to $t=72 \mathrm{~min}$, after the beginning of the observations. 
A\&A 533, A96 (2011)
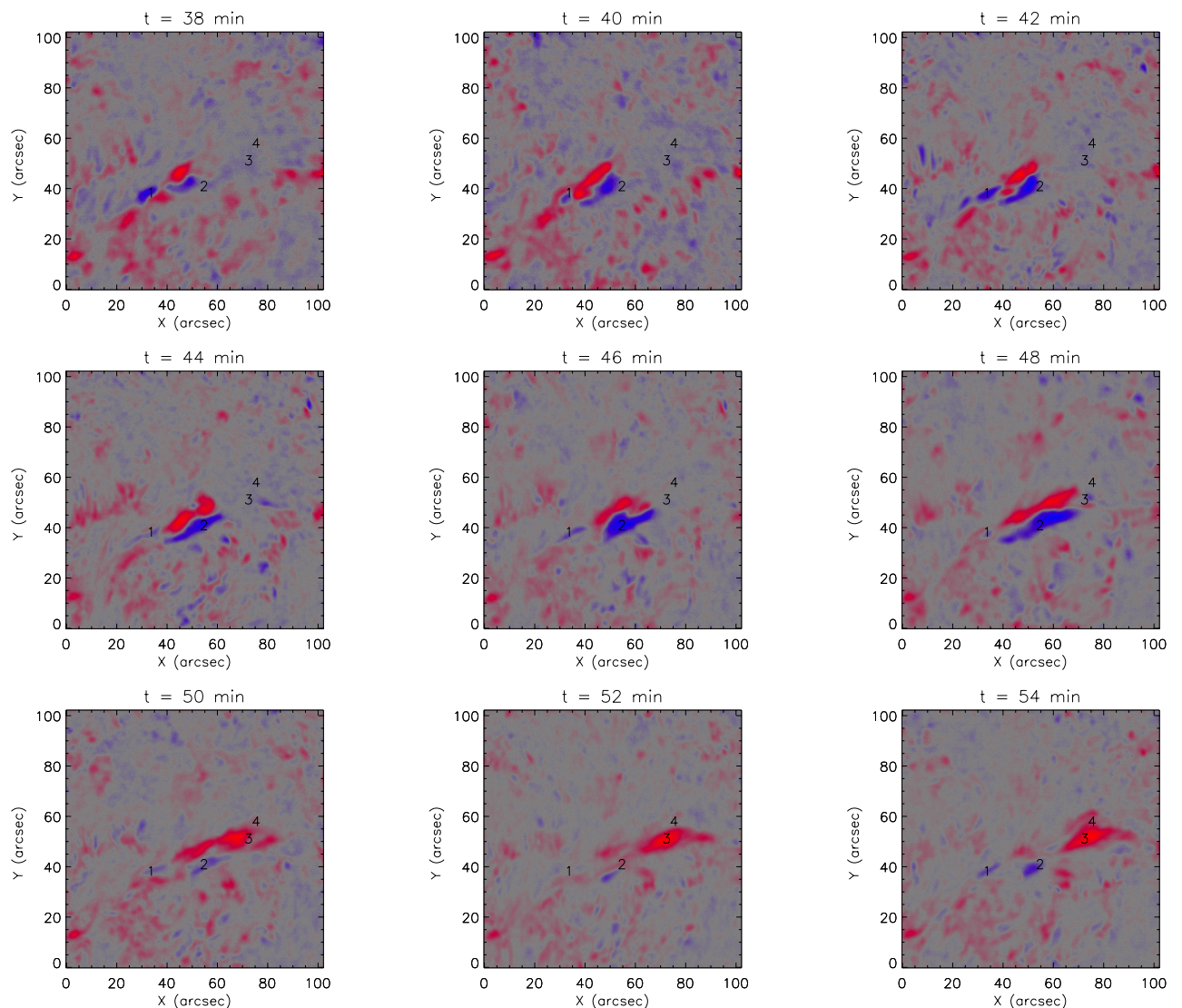

Fig. 15. Part of the velocity sequence in $\mathrm{H} \alpha$ from $t=38 \mathrm{~min}$ to $t=54 \mathrm{~min}$. Colours correspond to upward velocities in blue (up to $15 \mathrm{~km} \mathrm{~s}^{-1}$ ) and to downward velocities in red (up to $20 \mathrm{~km} \mathrm{~s}^{-1}$ ).
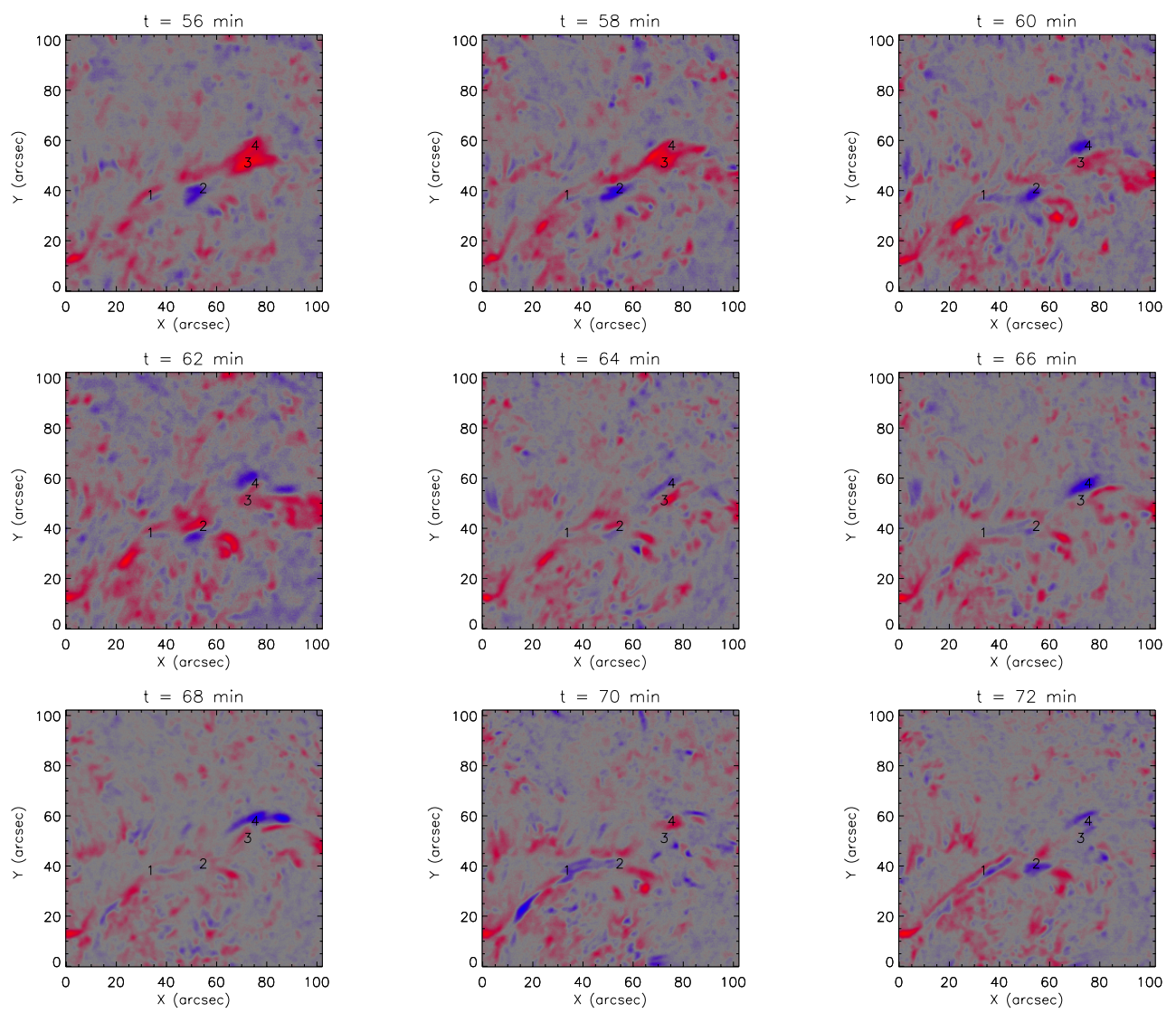

Fig. 16. Part of the velocity sequence in $\mathrm{H} \alpha$ from $t=56 \mathrm{~min}$ to $t=72 \mathrm{~min}$. Colours correspond to upward velocities in blue (up to $15 \mathrm{~km} \mathrm{~s}^{-1}$ ) and to downward velocities in red (up to $20 \mathrm{~km} \mathrm{~s}^{-1}$ ). 
K. Bocchialini et al.: Oscillatory motions observed in eruptive filaments
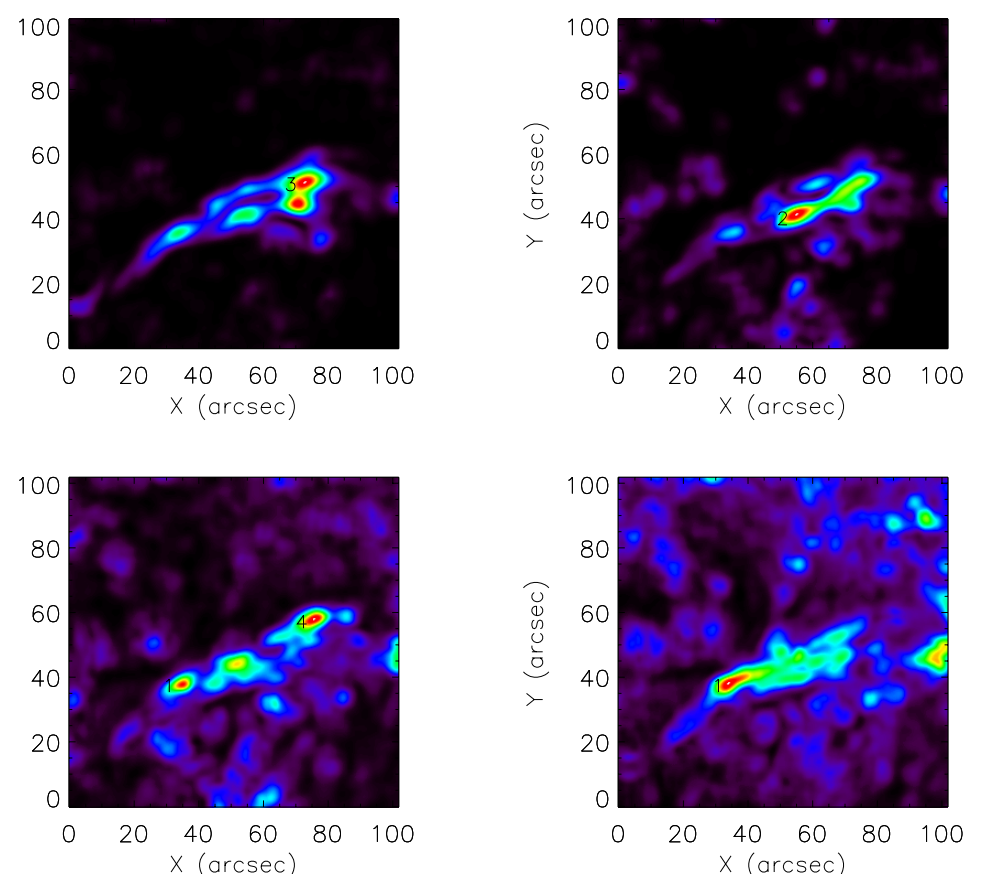

Fig. 17. Power maps of the velocity sequence in $\mathrm{H} \alpha$ from left to right and top to bottom, in the ranges [15, $55 \mathrm{~min}],[8,15 \mathrm{~min}]$, [4, $8 \mathrm{~min}]$, and $[2,4 \mathrm{~min}]$.
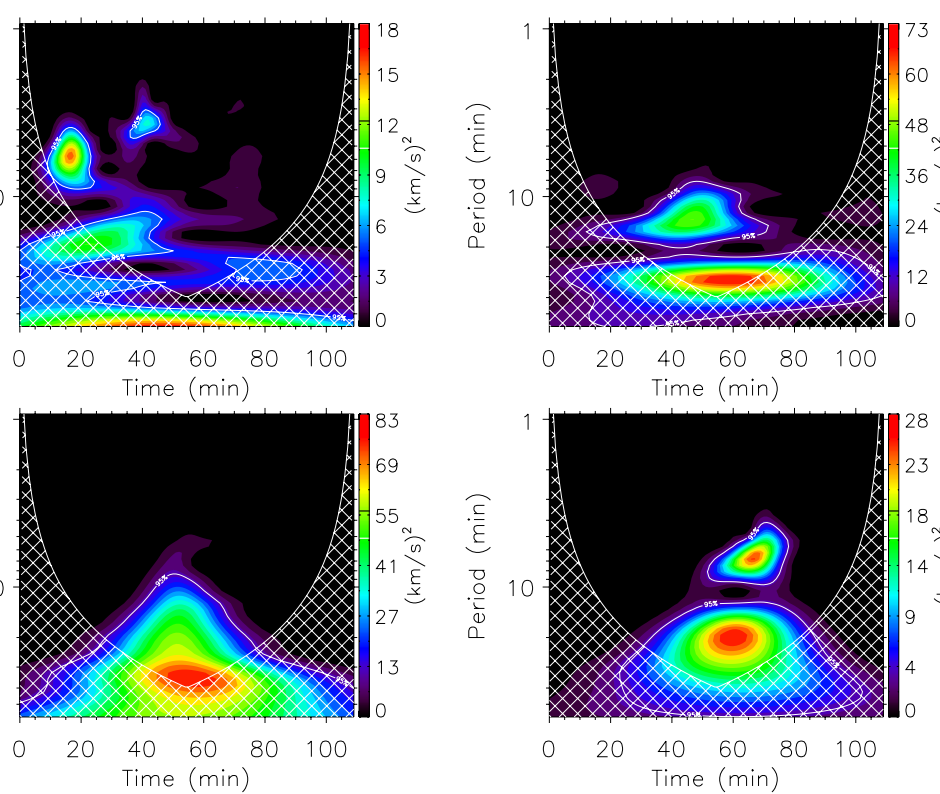

Fig. 18. Wavelet analysis of the velocity in $\mathrm{H} \alpha$ in zones 1 (top left), 2 (top right), 3 (bottom left), and 4 (bottom right); significant power is within the $95 \%$ confidence level contour and outside the hatched cones of influence.

and depends on the radiation time. Ballester (2006) points out in his review of solar corona seismology that other mechanisms are possible, such as wave leakage, resonant absorption and ionneutral collisions. Soler et al. (2007) and Soler et al. (2009) demonstrate that radiative effects of the prominence plasma are responsible for the attenuation of slow modes, connected to intermediate period (10 to $40 \mathrm{~min}$ ) prominence oscillations. The simultaneous upflows and downflows detected in the filament observed in the wings of $\mathrm{H} \alpha$ could be compared to the results obtained by Berger et al. (2010). High spatial resolution observations in $\mathrm{H} \alpha$ with Hinode/SOT of quiescent prominences reveal the presence of upflowing plumes. Their occurrence, combined with the plume dimensions, indicates that "the plumes are sources of upward mass flux into the prominence which partially offset the continual downward mass flux of heavy prominence material".

These results on small-amplitude oscillations of the intensity and of the Doppler velocity signals in eruptive filaments contribute to the statistical study needed to check the link between oscillations and eruption precursors.

Acknowledgements. The $\mathrm{H} \alpha$ images are courtesy of the Kiepenheuer Institute and NSO/Sacramento Peak. The EIT, CDS, and MDI data are courtesy of SOHO consortia. SOHO is a project of international cooperation between ESA and NASA. The Yohkoh/SXT images are from the JAXA consortium. The authors thank the anonymous referee for helpful comments and suggestions and L.M.R. Lock for his help. 


\section{References}

Abramenko, V. I., Wang, T., \& Yurchishin, V. B. 1996, Sol. Phys., 168, 75 Anzer, U. 2009, A\&A, 497, 521

Arregui, I., \& Ballester, J. 2010, Space Sci. Rev., 59

Ballester, J. 2006, Lecture Notes and Essays in Astrophysics, 2, 91

Baudin, F., Molowny-Horas, R., \& Koutchmy, S. 1997, A\&A, 326, 842

Baudin, F., Bocchialini, K., Delannee, C., et al. 1998, in New Perspectives on Solar Prominences, ed. D. F. Webb, B. Schmieder, \& D. M. Rust, IAU Colloq., 167, ASP Conf. Ser., 150, 314

Berger, T. E., Slater, G., Hurlburt, N., et al. 2010, ApJ, 716, 1288

Bocchialini, K., Costa, A., Domenech, G., et al. 2001, Sol. Phys., 199, 133

Chen, P. F., Innes, D. E., \& Solanki, S. K. 2008, A\&A, 484, 487

Delaboudinière, J.-P., Artzner, G., Brunaud, J., et al. 1995, Sol. Phys., 162, 291

Delbouille, L., Roland, G., \& Neven, L. 1973, Atlas photométrique du spectre solaire de $\lambda=3000$ à $\lambda=10$ 000, Université de Liège, Institut d'Astrophysique Engvold, O. 2008, in IAU Symp. 247, ed. R. Erdélyi, \& C. A. Mendoza-Briceño, 152

Filippov, B., \& Koutchmy, S. 2002, Sol. Phys., 208, 283

Filippov, B., \& Koutchmy, S. 2008, Ann. Geophys., 26, 3025

Fleck, B., Domingo, V., \& Poland, A. 1995, Sol. Phys., 162

Foullon, C., Verwichte, E., \& Nakariakov, V. 2004, A\&A, 427, L5

Foullon, C., Verwichte, E., \& Nakariakov, V. 2009, ApJ, 700, 1658

Georgakilas, A., Koutchmy, S., \& Christopoulou, E. 2001, A\&A, 370, 273

Harrison, R., Sawyer, E., Carter, M., et al. 1995, Sol. Phys., 162, 233

Isobe, H., \& Tripathi, D. 2006, A\&A, 449, L17

Isobe, H., Tripathi, D., Asai, A., \& Jain, R. 2007, Sol. Phys., 246, 89

Koutchmy, S., Slemzin, V., Filippov, B., et al. 2008, A\&A, 483, 599

Labrosse, N., Heinzel, P., Vial, J., et al. 2010, Space Sci. Rev., 151, 243

Mackay, D. H., Karpen, J. T., Ballester, J. L., Schmieder, B., \& Aulanier, G. 2010, Space Sci. Rev., 151, 333
Malherbe, J., Tarbell, T., Wiik, J. E., et al. 1997, ApJ, 482, 535

Molowny-Horas, R., Oliver, R., Ballester, J., \& Baudin, F. 1997, Sol. Phys., 172, 181

Molowny-Horas, R., Oliver, R., Ballester, J., \& Baudin, F. 1998, in New Perspectives on Solar Prominences, ed. D. F. Webb, B. Schmieder, \& D. M. Rust, IAU Colloq., 167, ASP Conf. Ser., 150, 139

Ning, Z., Cao, W., Okamoto, T. J., Ichimoto, K., \& Qu, Z. Q. 2009, A\&A, 499, 595

Okamoto, T. J., Tsuneta, S., Berger, T. E., et al. 2007, Science, 318, 1577

Oliver, R. 2009, Space Sci. Rev., 149, 175

Oliver, R., \& Ballester, J. L. 2002, Sol. Phys., 206, 45

Pintér, B., Jain, R., Tripathi, D., \& Isobe, H. 2008, ApJ, 680, 1560

Pouget, G., Bocchialini, K., \& Solomon, J. 2006, A\&A, 450, 1189

Priest, E., \& Forbes, T. 2000, Magnetic Reconnection, ed. E. Priest, \& T. Forbes

Ramsey, H., \& Smith, S. 1966, AJ, 71, 197

Régnier, S., Solomon, J., \& Vial, J. 2001, A\&A, 376, 292

Scherrer, P., Bogart, R., Bush, R., et al. 1995, Sol. Phys., 162, 129

Schmieder, B., Mein, P., \& Thompson, W. 1991, Adv. Space Res., 11, 195

Soler, R., Oliver, R., \& Ballester, J. 2007, A\&A, 471, 1023

Soler, R., Oliver, R., \& Ballester, J. 2009, New Astron., 14, 238

Terradas, J., Molowny-Horas, R., Wiehr, E., et al. 2002, A\&A, 393, 637

Terradas, J., Carbonell, M., Oliver, R., \& Ballester, J. 2005, A\&A, 434, 741

Thompson, W. 1999, CDS Software Note, 53

Torrence, C., \& Compo, G. 1998, Bull. Am. Meteorol. Soc., 79, 61

Tripathi, D., Isobe, H., \& Jain, R. 2009, Space Sci. Rev., 149, 283

Vrsnak, B. 1990, Sol. Phys., 129, 295

Vrsnak, B., Ruzdjak, V., Brajsa, R., \& Zloch, F. 1990, Sol. Phys., 127, 119

Vršnak, B., Veronig, A. M., Thalmann, J. K., \& Žic, T. 2007, A\&A, 471, 295

Wiehr, E., Balthasar, H., \& Stellmacher, G. 1989, Hvar Observatory Bulletin, 13,131 